Title : will be set by the publisher

Editors : will be set by the publisher

EAS Publications Series, Vol. ?, 2007

\title{
MICROSCOPIC DIFFUSION IN STELLAR PLASMAS
}

\author{
Thoul A. ${ }^{1}$ and Montalban J. ${ }^{1}$
}

\begin{abstract}
We review the basic equations describing the microscopic diffusion of elements in stars. We describe the two formalisms commonly used to describe a stellar plasma, i.e., the Chapman-Enskog method and the Burgers equations. We point out the underlying assumptions about the physical state of the plasma itself. We briefly describe the different approximations which are often used to solve these equations, and discuss their validity. One of the major problems lies in the calculation of the collision integrals, and we discuss the different approaches to solving these integrals, emphasizing on their domain of validity.
\end{abstract}

\section{Introduction}

We give an overview of the different formalisms which have been used by astrophysicists to describe stellar plasmas, without going into too many details. We do not intend here to give a complete bibliography on the subject of diffusion in stellar plasmas; the references which appear have been chosen amongst many others, and the reader can find more references on the subject in those papers. The literature dealing with this topic is however sometimes quite confusing, and we try here to point out the major points where it is necessary to be particularly attentive.

The methods described in this paper are based on the following assumptions: negligible radiative forces, complete ionisation, no magnetic fields, Maxwellian velocity distributions and same temperature for all ions and electrons, diffusion velocities much smaller than the mean thermal velocities, collisions dominated by classical interactions between two point particles, plasma can be considered as a dilute gas, i.e., the ideal gas equation of state applies.

Another review paper in this volume (Alecian 2007) is dedicated to the treatment and effects of the radiative forces. The effects of diffusion on the evolution and seismology of the Sun and of main sequence stars are reviewed in other papers of this volume (Christensen-Dalsgaard and Di Mauro 2007, Chaboyer 2007) .

\footnotetext{
${ }^{1}$ Department of Astrophysics and Geophysics, Liège University, 17 allée du 6 août, 4000 Liège, Belgium
} 
We start by some reminders about basic plasma physics and the concept of Debye shielding. We then give a brief presentation of the two formalisms commonly used to describe transport processes in stellar plasmas, namely the ChapmanEnskog and the Burgers approaches. We then give a review of the existing formulae and numerical results that can be found in the literature for the collision integrals.

\section{Some basics on plasma physics: Debye shielding and the Debye- Hückel potential}

A plasma is defined as "a gas of charged particles in which the potential energy of a typical particle due to its nearest neighbours is much smaller than its kinetic energy" (see, e.g., Nicholson 1983). Another definition often found is that a plasma is "a quasineutral gas of charged particles which exhibits collective behaviour". These two definitions are in fact equivalent, as we briefly show now. by

Consider a test charge particle $Q$ in a plasma. The Poisson equation is given

$$
\nabla^{2} \phi=-4 \pi \rho_{e}=4 \pi e\left(n_{e}-\sum_{i} Z_{i} n_{i}\right)
$$

where $\phi$ is the electrostatic potential, $\rho_{e}$ is the charge density, $n_{e}$ and $n_{i}$ are the electron and ions number densities, and $Z_{i}$ is the charge of ion $i$. At equilibrium

$$
\begin{array}{r}
n_{i}=n_{0 i} e^{-Z_{i} e \phi / k_{B} T} \\
n_{e}=n_{0 e} e^{e \phi / k_{B} T}
\end{array}
$$

where $k_{B}$ is Boltzmann's constant and we assumed that ions and electrons have the same temperature $T$.

If the particles kinetic energy is much larger than the potential energy, i.e., if $e \phi \ll k_{B} T$ then

$$
\begin{array}{r}
n_{i} \approx n_{0 i}\left(1-Z_{i} e \phi / k_{B} T\right) \\
n_{e} \approx n_{0 e}\left(1+e \phi / k_{B} T\right)
\end{array}
$$

and Poisson's equation can be rewritten as

$$
\nabla^{2} \phi \approx \frac{4 \pi e^{2}}{k_{B} T}\left(n_{0 e}+\sum_{i} n_{0 i} Z_{i}^{2}\right) \phi=\lambda_{D}^{-2} \phi
$$

where we have assumed that the gas is quasineutral, i.e., $n_{0 e}-\sum_{i} Z_{i} n_{0 i} \approx 0$ and

$$
\lambda_{D}=\left(\frac{k_{B} T}{4 \pi e^{2} \sum_{s} Z_{s}^{2} n_{s}}\right)^{1 / 2}
$$

the sum over $s$ being over all species, including electrons. The solution of equation (2.6) is given by

$$
\phi=\frac{Q}{r} e^{-r / \lambda_{D}}
$$


In a plasma the potential due to a test charge falls off much faster than in vacuum. The electrostatic potential is "shielded" on a distance of the order of the Debye length $\lambda_{D}$. This is called the "Debye shielding".

It is easy to verify that the condition $e \phi \ll k_{B} T$ is equivalent to $\Lambda_{P}=n_{0} \lambda_{D}^{3} \gg$ 1 , i.e., for a ionised gas to be a plasma there must be many particles in a Debye sphere.

Table 1. Typical parameters for different plasmas

\begin{tabular}{|c|c|c|c|c|c|c|}
\hline & $\mathrm{T}$ & $\rho$ & $\mathrm{n}$ & $\mathrm{a}_{0}$ & $\lambda_{D}$ & $\Lambda_{P}$ \\
\hline & $\mathrm{K}$ & $\mathrm{g} / \mathrm{cm}^{3}$ & $\mathrm{~cm}^{-3}$ & $\mathrm{~cm}$ & $\mathrm{~cm}$ & \\
\hline Thermonuclear reactor & $10^{8}$ & & $10^{15}$ & & $10^{-3}$ & $10^{6}$ \\
\hline Interstellar gas & $10^{4}$ & & 1 & & $10^{3}$ & $10^{8}$ \\
\hline Solar corona & $10^{6}$ & & $10^{9}$ & & 0.2 & $10^{7}$ \\
\hline Solar atmosphere & $10^{4}$ & & $10^{14}$ & & $10^{-4}$ & 40 \\
\hline Solar convective zone & $4 \times 10^{6}$ & 1 & $6 \times 10^{23}$ & $7 \times 10^{-9}$ & $10^{-8}$ & 1 \\
\hline Solar centre & $1.5 \times 10^{7}$ & 150 & $10^{26}$ & $10^{-9}$ & $10^{-9}$ & 1 \\
\hline
\end{tabular}

In astrophysics papers, the plasma parameter is often defined as

$$
\Lambda=\frac{e^{2}}{k_{B} T \lambda_{D}}\left(\frac{\sum_{i} n_{i} Z_{i}^{2}}{\sum_{i} n_{i}}\right)
$$

which, to a factor $4 \pi$, is the inverse of $\Lambda_{P}$. Therefore, in astronomy papers, an ionised gas is a plasma if $\Lambda \ll 1$. Plasmas with $\Lambda \ll 1$ are called "dilute plasmas" or "weakly coupled plasmas".

In a dilute plasma, an excellent approximation of the interaction between two classical particles of charges $Z_{s}$ and $Z_{t}$ is given by the static screened Coulomb potential, also called the Debye-Hückel potential

$$
V_{s t}(r)=Z_{s} Z_{t} e^{2} \frac{e^{-r / \lambda_{D}}}{r}
$$

As long as $\Lambda \ll 1$, the Boltzmann equation is rigorously valid, and the ideal gas equation of state applies.

Typical values of the temperature $T$, the mass density $\rho$, the number density $n$, the interionic distance $a_{0}$, the Debye length $\lambda_{D}$, and the plasma parameter $\Lambda_{P}$ are given in Table 1 for different plasmas. Plasmas present in thermonuclear reactors, the interstellar gas, the solar corona, and the solar atmosphere are obviously weakly coupled plasmas, and the "classical plasma theory" applies. However, in the solar interior the plasma parameter is of order 1 , and the classical concept of the Debye length, shielding, and collective behaviour of the particles is no longer strictly valid. It is customary however to extend the results obtained in the weakly coupled regime to denser plasmas.

According to Monchick (1985) the inclusion of quantum effects and dynamical shielding reduces the diffusion coefficient in the Sun by about 40-50\%, most of it due to dynamical shielding. 


\section{Descriptions of stellar plasmas}

Transport coefficients in stellar plasmas are evaluated in the limit of the dilutegas approximation. The collisions are treated classically and transport equations are obtained from approximate solutions of the Boltzmann equation for binary or multiple gas mixtures:

$$
\frac{\partial f_{i}}{\partial t}+\vec{v}_{i} \cdot \frac{\partial f_{i}}{\partial \vec{r}}+\vec{F}_{i} \cdot \frac{\partial f_{i}}{\partial \vec{v}_{i}}=\frac{\partial_{e} f_{i}}{\partial \vec{v}_{i}}
$$

where $f_{i}=f_{i}(\vec{r}, \vec{v}, t)$ is the distribution function of species $i, \vec{F}_{i}$ are the external forces per unit mass, and the right-hand side represents the binary collision term.

Two different formalisms have been commonly used, those based on the ChapmanEnskog theory (Chapman \& Cowling 1970), and those based on Burgers' theory (Burgers 1969). In both methods, the diffusion coefficients can be written as functions of the collision integrals, which depend on the exact nature of the interaction between colliding particles.

A good discussion of the domain of validity of those methods can be found in Paquette \& al. (1986, hereafter P86).

\subsection{Descriptions based on Chapman-Enskog's theory}

The Chapman-Enskog theory (Chapman \& Cowling 1970) assumes that the total distribution function of a given species can be written as a convergent series, each term in the series representing a successive approximation to the distribution function:

$$
f_{i}(\vec{r}, \vec{v}, t)=f_{i}^{(0)}(\vec{r}, \vec{v}, t)+f_{i}^{(1)}(\vec{r}, \vec{v}, t)+f_{i}^{(2)}(\vec{r}, \vec{v}, t)+\ldots
$$

with

$$
\left|f_{i}^{(n)}\right| \ll\left|f_{i}^{(n-1)}\right|
$$

Substituting into Boltzman's equation and linearising gives a series of equations for each $f_{i}^{(n)}$ in terms of the lower order approximations $f_{i}^{(n-1)}, f_{i}^{(n-2)}, \ldots$ The lowestorder approximation $f_{i}^{(0)}$ is a Maxwellian distribution function characterised by $n_{i}, T$, and $v_{0}$. The transport coefficients are obtained by taking moments of the first-order approximation of the distribution function.

Good estimates of the transport coefficients are given by the so-called "firstorder" and "second-order approximations" to the transport properties, obtained by expanding the first-order distribution function on the basis of Soline polynomials, which gives a very rapidly convergent series. For a binary mixture, the diffusion velocity can be written as (Chapman \& Cowling 1970):

$$
\begin{aligned}
& v_{2}-v_{1}= \\
& \quad-D_{12}\left\{\frac{n^{2}}{n_{1} n_{2}} \nabla\left(\frac{n_{1}}{n_{2}}\right)+\frac{m_{1}-m_{2}}{\mu} \nabla \ln p+\frac{n^{2}}{n_{1} n_{2}} \frac{D_{t h}}{D_{12}} \nabla \ln T-\frac{m_{1} m_{2}}{\mu k_{B} T}\left(F_{2}-F_{1}\right)\right\}(3.4
\end{aligned}
$$


where $D_{12}$ is the molecular diffusion coefficient, $D_{t h}$ is the thermal diffusion coefficient, $n_{1}$ and $n_{2}$ are the number densities of the two gases, $n=n_{1}+n_{2}, m_{1}$ and $m_{2}$ are their atomic masses, $\mu$ is the reduced mass, $F_{1}$ and $F_{2}$ are external forces on atoms 1 and 2 .

In the atom-test approximation $\left(n_{2} \ll n_{1}\right)$ this expression reduces to

$$
v_{2}=-D_{12}\left\{\nabla \ln c+\left(1-\frac{m_{2}}{m_{1}}\right) \nabla \ln p+\alpha_{T} \nabla \ln T-\frac{m_{2}}{k_{B} T}\left(F_{2}-F_{1}\right)\right\}
$$

where $c=n_{2} / n$ and $\alpha_{T}=(1 / c)\left(D_{t h} / D_{12}\right)$. For a discussion on these expressions, see, e.g., Vauclair \& Vauclair 1982.

The diffusion coefficients $D_{12}$ and $D_{t h}$ are functions of the collision integrals, which will be discussed in Section 4 .

\subsection{Descriptions based on Burgers' equations}

The method developed by Burgers (1969) is based on the Grad 13 moment approximation and the use of a Fokker-Planck collision term in the Boltzmann equation.

The main advantage of Burgers's formalism is that it provides a more convenient way to treat multicomponent gases. In Burgers's formulation, all species are treated symmetrically, so the equations are valid for any number of constituents.

Burgers's equations are given by:

- the mass conservation equation

$$
\frac{\partial n_{i}}{\partial t}+\frac{1}{r^{2}} \frac{\partial}{\partial r}\left(n^{2} n_{i} w_{i}\right)=\left(\frac{\partial n_{i}}{\partial t}\right)_{n u c l},
$$

- the momentum conservation equation

$$
\frac{\partial p_{i}}{\partial r}+\rho_{i} g-\rho_{e i} E=\sum_{j \neq i}\left[K_{i j}\left(w_{j}-w_{i}\right)+z_{i j} K_{i j} \frac{m_{j} r_{i}-m_{i} r_{j}}{m_{i}+m_{j}}\right],
$$

and the energy conservation equation

$$
\begin{aligned}
\frac{5}{2} n_{i} k_{B} & \frac{d T}{d r}=-\frac{5}{2} \sum_{j \neq i} K_{i j} z_{i j} \frac{m_{i}}{m_{i}+m_{j}}\left(w_{j}-w_{i}\right)-\frac{2}{5} K_{i i} z_{i j}^{\prime \prime} r_{i} \\
& -\sum_{j \neq i} \frac{K_{i j}}{\left(m_{i}+m_{j}\right)^{2}}\left(3 m_{i}^{2}+m_{j}^{2} z_{i j}^{\prime}+0.8 m_{i} m_{j} z_{i j}^{\prime \prime}\right) r_{i} \\
& +\sum_{j \neq i} K_{i j} \frac{m_{i} m_{j}}{\left(m_{i}+m_{j}\right)^{2}}\left(3+z_{i j}^{\prime}-0.8 z_{i j}^{\prime \prime}\right) r_{j} .
\end{aligned}
$$

In these equations, $p_{i}$ is the gas pressure of species $i, \rho_{i}$ is its mass density, $\rho_{e i}$ is its charge density, $E$ is the electric field, $g$ is the gravitational acceleration, $w_{i}$ are the diffusion velocities, $r_{i}$ are the so-called "residual heat flow vectors", and $K_{i j}$, $z_{i j}, z_{i j}^{\prime}$, and $z_{i j}^{\prime \prime}$ are the resistance coefficients. 
The resistance coefficients are related to the collision integrals:

$$
\begin{gathered}
K_{i j}=\frac{16}{3} n_{i} n_{j} \frac{m_{i} m_{j}}{m_{i}+m_{j}} \Omega_{i j}^{(11)} \\
z_{i j}=1-\frac{2}{5} \frac{\Omega_{i j}^{(12)}}{\Omega_{i j}^{(11)}} \\
z_{i j}^{\prime}=\frac{5}{2}-\frac{\left.10 \Omega_{i j}^{(12}\right)-2 \Omega_{i j}^{(13)}}{5 \Omega_{i j}^{(11)}}
\end{gathered}
$$

and a similar expression for $z_{i j}^{\prime \prime}$. Expressions for the collision integrals can be found, e.g., in Michaud \& Proffitt (1993, hereafter MP93). In addition there are constraints due to local mass conservation

$$
\sum_{i} m_{i} n_{i} w_{i}=0
$$

current neutrality

$$
\sum_{i} q_{i} n_{i} w_{i}=0
$$

and charge neutrality

$$
\sum_{i} q_{i} n_{i}=0
$$

The Burgers equations with the constraints form a closed system of equations for the diffusion velocities $w_{i}$, the residual heat flow vectors $r_{i}$, the gravitational acceleration $g$, and the electric field $E$, in terms of the pressure $p$, the temperature $T$, and the concentration gradients $C_{i}=n_{i} / n_{e}$. Although it is long and tedious, this system can in principle be solved analytically. In practice, it is either solved numerically, or it is simplified using additional approximations.

An approximate solution to Burgers's equations can be obtained easily if the heat fluxes are neglected, $r_{i}=0$. In that case, the energy equation becomes unnecessary and the equation of momentum conservation becomes much easier to solve:

$$
\frac{\partial p_{i}}{\partial r}+\rho_{i} g-\rho_{e i} E=\sum_{j \neq i}\left[K_{i j}\left(w_{j}-w_{i}\right)\right]
$$

For a pure Hydrogen-Helium-electrons plasma, ignoring the collisions with the electrons, and assuming $m_{e} / m_{i} \ll 1$ the solution is

$$
w_{H}=\frac{k_{B} T n_{H}}{K_{H H_{e}}} \frac{1-X}{1-2 X}\left[\frac{5}{4}(1-X) \frac{d \ln p}{d r}+\frac{(X+3)}{(X+1)(5 X+3)} \frac{d \ln X}{d r}\right]
$$

where $X$ and $Y$ are the hydrogen and helium mass fractions and $p$ is the total pressure. This is identical to the simplified formula obtained for fully ionised H-He mixtures by MP93 (see equation (14) in their paper) if $Y \ll X$. 
The system of equations (3.6)-(3.8) and (3.12)-(3.14) can also be solved numerically, with no approximation. A Fortran routine (Thoul \& al. 1994, hereafter TBL94) which solves the Burgers equations is freely available at the following web sites:

http://www.sns.ias.edu/ jnb/

(follow the following links: solar neutrinos, software and data, diffusion subroutine) and

http://www.astro.ulg.ac.be/orientation/asterosis/article/article_e.html

The equations solved in this routine are the Burgers equations obtained using resistance coefficients $z_{i j}=0.6, z_{i j}^{\prime}=1.3, z_{i j}^{\prime \prime}=2$, calculated with a truncated Coulomb potential (see Section 4):

$$
\begin{gathered}
\frac{\partial p_{i}}{\partial r}+\rho_{i} g-\rho_{e i} E=\sum_{j \neq i} K_{i j}\left[\left(w_{j}-w_{i}\right)+0.6\left(x_{i j} r_{i}-y_{i j} r_{j}\right)\right] \\
\frac{5}{2} n_{i} k_{B} \frac{d T}{d r}=\sum_{j \neq i} K_{i j}\left\{\frac{3}{2} x_{i j}\left(w_{j}-w_{i}\right)-y_{i j}\left[1.6 x_{i j}\left(r_{i}+r_{j}\right)+Y_{i j} r_{i}-4.3 x_{i j} r_{j}\right]\right\}
\end{gathered}
$$

where $x_{i j}=\mu_{i j} / m_{i}, y_{i j}=\mu_{i j} / m_{j}, Y_{i j}=3 y_{i j}+1.3 x_{i j} m_{j} / m_{i}$ and

$$
K_{i j}=\frac{2^{3 / 2} \sqrt{\pi}}{3} e^{4} Z_{i}^{2} Z_{j}^{2} n_{i} n_{j}\left(\frac{m_{i} m_{j}}{m_{i}+m_{j}}\right)^{1 / 2}\left(k_{B} T\right)^{-3 / 2} 2 \ln \Lambda_{i j} .
$$

In TBL94's routine, the Coulomb logarithms $\ln \Lambda_{i j}$ are those given by Iben \& MacDonald (85). Please note a typo in equation 9 of TBL94, which should read

$$
\ln \Lambda_{s t}=\frac{1.6249}{2} \ln \left[1+0.18769\left(\frac{4 k_{B} T \lambda}{Z_{s} Z_{t} e^{2}}\right)^{1.2}\right]
$$

\section{The resistance coefficients}

Whichever of the two methods is used to obtain the transport coefficients, one of the main difficulties lies in the calculation of the collision integrals. Those integrals diverge when a pure Coulomb potential is used. The easiest solution to the convergence problem is to use a pure Coulomb potential for the interactions between charged particles, with a long-range cutoff at the Debye length $\lambda_{D}$. In this case, the collision integrals can be calculated analytically. A better, more realistic solution is to use a shielded Debye-Hückel potential of the form (see equation 2.10):

$$
V_{i j}=\frac{Z_{i} Z_{j} e^{2}}{r} e^{-r / \lambda_{D}}
$$


As explained in Section 2, this interaction potential is only valid if there are many particles in a Debye sphere. In dense plasmas this is no longer the case and it is better then to replace the Debye length $\lambda_{D}$ by the interionic distance $a_{o}$, with

$$
a_{0}=\left(\frac{3}{4 \pi \sum_{\text {ions }} n_{i}}\right)^{1 / 3} .
$$

A good solution is to use the so-called modified Debye-Hückel potential given by

$$
V_{i j}=\frac{Z_{i} Z_{j} e^{2}}{r} e^{-r / \max \left(\lambda_{D}, a_{0}\right)}
$$

Finally, quantum corrections can be added (Schlattl and Salaris 2003).

If a truncated Coulomb potential is used, the collision integrals can be calculated analytically. This gives $z_{i j}=0.6, z_{i j}^{\prime}=1.3, z_{i j}^{\prime \prime}=2$ (see, e.g., P86).

Numerical solutions have been obtained by P86 for the resistance coefficients $z_{i j}, z_{i j}^{\prime}, z_{i j}^{\prime \prime}$, calculated using a modified Debye-Hückel potential. The results for $z_{i j}, z_{i j}^{\prime}, z_{i j}^{\prime \prime}$, are plotted in figure (3) of MP93. At low densities, the results agree with those obtained using a truncated pure Coulomb potential, but differ at higher densities. In that regime, they agree well with Monte-Carlo results obtained by Hansen (1978) using as theoretical model a one-component plasma in which classical ions interact strongly in a uniform non-interactive neutralising background of degenerate electrons. P86 argue that since their results agree with those obtained in the two extreme regimes $(\Lambda \ll 1$ and $\Lambda \gg 1)$ they are probably also quite good in the intermediate regime $(\Lambda \sim 1)$, although they admit that there is no physical justification for this to be true. The numerical results of P86 were fitted by MP93, and more accurate calculations were performed by MacDonald (1991) at high densities using the same formalism.

There are many different approximations and fits for the Coulomb logarithm $\ln \Lambda_{i j}$. The value used in TBL94's routine is a fit by Iben \& MacDonald (1985) of numerical results obtained by Fontaine \& Michaud (1979) using a modified Debye-Hückel potential at low densities and a Thomas-Fermi potential at high densities:

$$
2 \ln \Lambda_{i j}=1.6249 \ln \left[1+0.18769\left(\frac{4 k_{B} T \lambda}{Z_{i} Z_{j} e^{2}}\right)^{1.2}\right]
$$

where $\lambda=\max \left(\lambda_{\mathrm{D}}, \mathrm{a}_{0}\right)$.

MP93 replace the Coulomb logarithm by $C_{i j}$ which is a function of $\left(2 k_{B} T \lambda / Z_{i} Z_{j} e^{2}\right)$ :

$$
K_{i j}=\frac{2^{3 / 2} \sqrt{\pi}}{3} e^{4} Z_{i}^{2} Z_{j}^{2} n_{i} n_{j}\left(\frac{m_{i} m_{j}}{m_{i}+m_{j}}\right)^{1 / 2}\left(k_{B} T\right)^{-3 / 2} C_{i j} .
$$

They showed that using $\ln \Lambda_{i j}$ as an approximation for $C_{i j}$ is valid in the low density limit. It is important to note that there is a factor 2 difference in the 
definition of $\ln \Lambda_{i j}$ in different papers. In particular, $\ln \Lambda_{i j}$ in TBL94 is different from $\ln \Lambda_{i j}$ in MP93, with

$$
\left(2 \ln \Lambda_{i j}\right)_{T B L 94}=\left(\ln \Lambda_{i j}\right)_{M P 93} .
$$
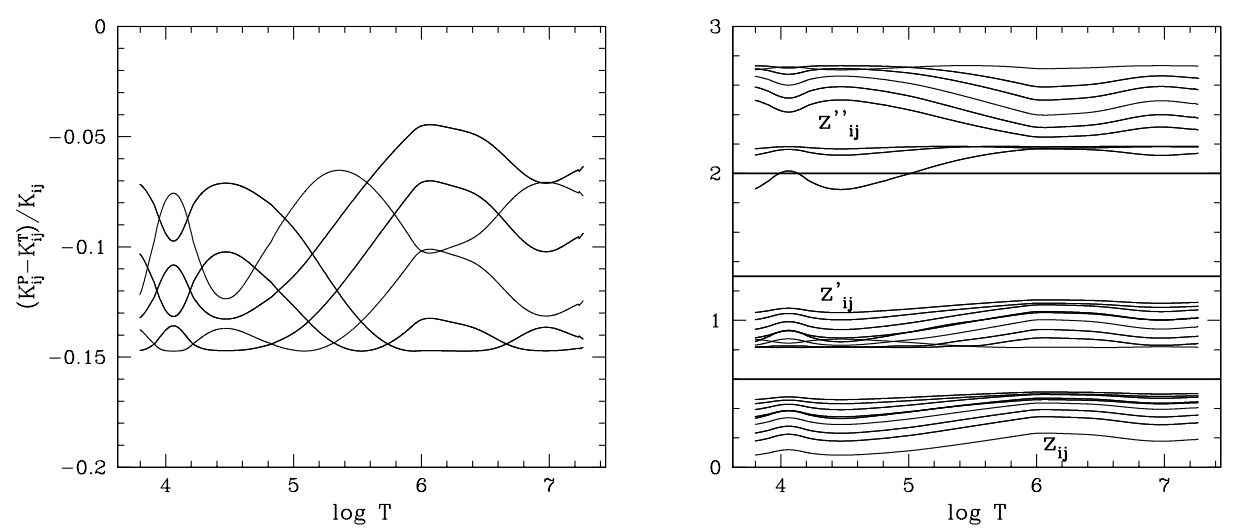

Fig. 1. Left panel: difference between friction coefficients calculated by TBL94's routine $\left(K_{\mathrm{ij}}^{\mathrm{T}}\right)$ and those calculated using P86's resistance coefficients as done in CESAM2k B $\left(K_{\mathrm{ij}}^{\mathrm{P}}\right)$, for the internal structure of the CLES Task3.2 A's model. The elements $i$ and $j$ are $\mathrm{H}, \mathrm{He}, \mathrm{Z}$ (all fully ionised) and electrons. Right panel: the heat flux terms $z_{\mathrm{ij}}$, $z_{\mathrm{ij}}^{\prime}$ and $z_{\mathrm{ij}}^{\prime \prime}$ calculated in CESAM using P86's results, for the same stellar structure and species as in left panel. The horizontal lines corresponds to the low density asymptotic values adopted in TBL94's routine.

In the low density limit, neglecting the heavy elements in the calculation of the Debye length, and assuming that the electrons are non-degenerate, it is easy to show that $\ln \Lambda_{X Y}$ can be written as (see, e.g., MP93):

$$
\ln \Lambda_{X Y}=-19.95-\frac{1}{2} \ln \rho+\frac{3}{2} \ln T-\frac{1}{2} \ln \frac{X+3}{2}
$$

where $X$ and $Y$ are the Hydrogen and Helium mass fractions.

P86 showed that their result for the self-diffusion of Helium agrees perfectly in a dilute plasma with that obtained with a truncated pure Coulomb potential, but is very different at high densities. Their fit is however the closest approximate solution to the "exact" solution at high densities.

Iben et al. (1992) compare the fits by Iben and MacDonald (1985) with those of P86. They conclude that they are very close, except at high densities.

Schlattl and Salaris (2003) introduced quantum corrections. They compared several classical calculations, showing that they are all very close, and they compared their quantum results with the classical ones for the Sun. The corrections 
are largest for ion-electron collisions, which give a small contribution to the diffusion coefficients. The quantum corrections on the ion-ion collisions are more pronounced at higher densities, as expected. They compared the results obtained for the sound speed in the Sun using different prescriptions for the diffusion coefficients. They show that the results obtained using the most accurate resistance coefficients with quantum corrections are very close, by chance, to those obtained using TBL94's routine.

\section{Example: Task 3.2 Comparisons for the resistance coefficients and the diffusion velocities}

For a selected stellar structure (Task3.2A computed with CLES, see Montalban $\&$ al. 2007 in this volume) we have compared the values of the coefficients $K_{i j}$,

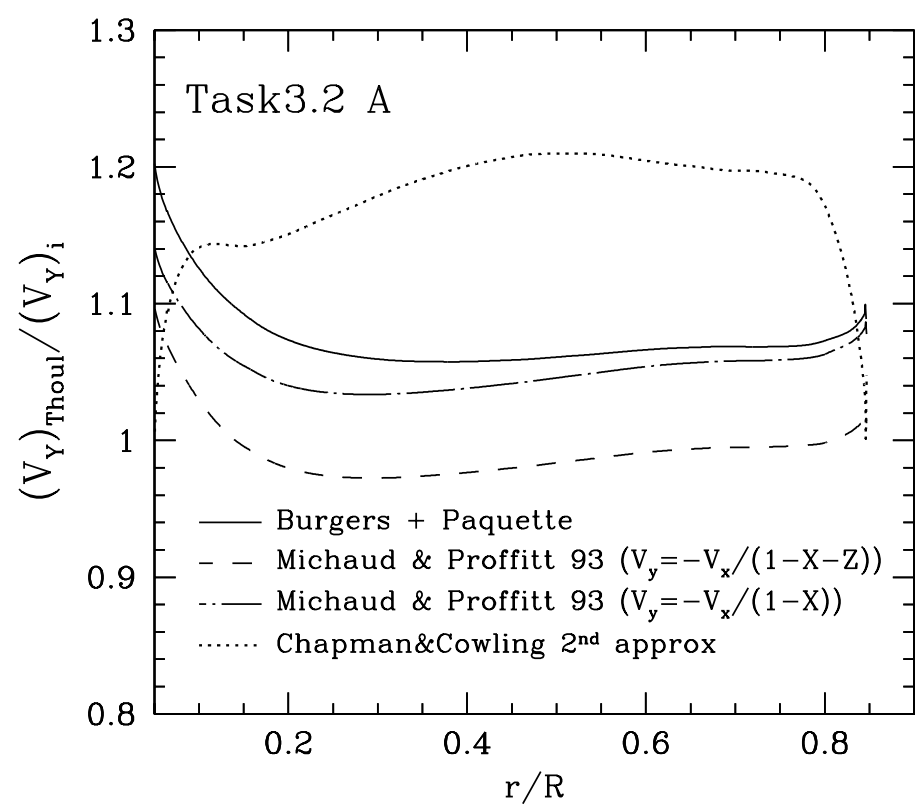

Fig. 2. Ratio between the helium diffusion velocity for the Task3.2 A' model derived from TBL94 and the one obtained using Michaud \& Proffitt (1993)'s formalism (dashed and dot-dashed lines), the one obtained from Burgers' equations and P86's collision integrals (solid line), and those obtained from Chapman \& Cowling approximation (dotted line) 
$z_{i j} z_{i j}^{\prime}$ and $z_{i j}^{\prime \prime}$ ( $i$ and $j$ being fully ionised $\mathrm{H}, \mathrm{He}, \mathrm{Z}$, and electrons) provided by TBL94's routine with those provided by the CESAM routine, which uses the P86's results. The comparisons are plotted in Fig. 1. We see that for this stellar model, the resistance coefficients from TBL94's routine $\left(K_{i j}^{\mathrm{T}}\right)$ are larger by 5 to $15 \%$ than those provided by P86's calculations $\left(K_{i j}^{\mathrm{P}}\right)$. The heat flux terms are also quite different from their asymptotic values (horizontal lines) as adopted in TBL94.

We have also computed the He diffusion velocity $\left(V_{\mathrm{Y}}\right)$ for the same stellar structure and for five different approaches: TBL94, TBL94 with P86's coefficients, MP93 (their Eqs. 17 - 19), and the Chapman \& Cowling approximation (eqs. (5)(8) in P86). Their relative values are plotted in Fig. 2. We see that the TBL94's calculations overestimate the diffusion velocity by 6 to $20 \%$ (at the stellar centre) with respect to the computations with P86's coefficients.

\section{Conclusions}

The descriptions of stellar plasmas are all based on the Boltzmann equation, and are therefore valid for classical plasmas, i.e., plasmas with $\Lambda \ll 1$. However in the interior of main-sequence stars, the plasma parameter is of the order 1 , and the Boltzmann equation is no longer strictly valid. No physically correct theory exists in that regime.

There are two families of descriptions of stellar plasmas: those based on the Chapman-Enskog theory, and those based on Burgers' theory. The Burgers' theory is equivalent to the second-order approximation in the Chapman \& Cowling approximation. They are valid for weakly coupled plasmas. More accurate results could be obtained with higher-order approximations in the Chapman-Cowling formalism, but these are intractable for multicomponent gases. The Burgers equation have the advantage to be the easiest to use for multicomponent gases.

Independently of the description used, there remains the problem of the calculation of the collision integrals and thus the resistance coefficients. These quantities are easy to calculate in weakly coupled plasmas, where a simple pure Coulomb potential truncated at the Debye length gives very good results. A shielded potential gives more accurate results, but these are also valid only for weakly coupled plasmas. A modified Debye-Hückel potential gives good results up to high densities, even though there are no real physical arguments to justify this result. Finally, recent quantum calculations have been made available.

\section{AKNOWLEDGMENTS}

Anne Thoul is Chercheur Qualifié FNRS. Josefina Montalban is supported by PRODEX Contract number C90197. Both received financial support from HELAS.

\section{References}

Alecian, G. 2007, these proceedings, EAS Publication Series

Burgers, J.M. 1969, "Flow equations for Composite Gases", New York: Academic Press 
Chapman, S. and Cowling, T.G. 1970, "The mathematical theory of non-uniform gases An account of the kinetic theory of viscosity, thermal conduction and diffusion in gases", Cambridge: University Press

Chaboyer, B. 2007, EAS Publication series

Christensen-Dalsgaard, J. and Di Mauro, P. 2007, these proceedings, EAS Publication series

Fontaine, G. and Michaud, G. 1979, in "White dwarfs and variable degenerate stars", Proceedings of the Colloquium, and Proceedings of the Fourth Annual Workshop on Novae, Dwarf Novae and Other Cataclysmic Variables, Rochester, N.Y., University of Rochester, p. 192

Hansen, J.P. 1978, in "Strongly Coupled Plasmas". Edited by Gabor Kalman, and Paul Carini. D. Reidel Publishing Company, Dordrecht-Holland. NATO Advanced Study Institutes Series. Volume B36, p.117

Iben, I. Jr. and MacDonald, J. 1985, ApJ, 296, 540

Iben, I. Jr., Fujimoto,M.Y. \& MacDonald, J. 1992, AJ, 388, 521

MacDonald, J. 1991, ApJS, 76, 369

Michaud, G. and Proffitt, C.R. 1993,in "Inside the stars", Proceedings of the 137th IAU Colloquium, Univ. of Vienna, Austria, p. 246 (MP93)

Monchick, L. 1985, Phys. Fluids, 28, 3341

Montalban, J., Théado, S. and Lebreton, Y. 2007, these proceedings, EAS Publication series

Nicholson, D.R. 1983, "Introduction to Plasma Theory", John Wiley \& Sons: New York Paquette, C., Pelletier, C., Fontaine, G. and Michaud, G. 1986, ApJS, 61, 177 (P86)

Schlattl, H. and Salaris, M. 2003, A\&A, 402,29

Thoul, A.A., Bahcall, J.N., Loeb, A. 1994, ApJ, 421, 828 (TBL94)

Vauclair, S. and Vauclair, G. 1982, ARA\&A, 20, 37 